Pacific Journal of Mathematics

EXTENSIONS OF THEOREMS OF CUNNINGHAM-AIGNE 


\section{EXTENSIONS OF THEOREMS OF CUNNINGHAM-AIGNER AND HASSE-EVANS}

\section{Richard H. Hudson and KeNNETh S. Williams}

If $k$ is a positive integer and $p$ is a prime with $p \equiv 1\left(\bmod 2^{k}\right)$, then $2^{(p-1) / 2^{k}}$ is a $2^{k}$ th root of unity modulo $p$. We consider the problem of determining $2^{(p-1) / 2^{k}}$ modulo $p$. This has been done for $k=1,2,3$ and the present paper treats $k=4$ and 5 , extending the work of Cunningham, Aigner, Hasse, and Evans.

1. Introduction. When $k=1$, we have the familiar result

$$
2^{(p-1) / 2} \equiv \begin{cases}+1(\bmod p), & \text { if } p \equiv 1,7(\bmod 8), \\ -1(\bmod p), & \text { if } p \equiv 3,5(\bmod 8) .\end{cases}
$$

When $k=2$ and $p \equiv 1(\bmod 4)$, there are integers $a \equiv 1(\bmod 4)$ and $b \equiv 0(\bmod 2)$ such that $p=a^{2}+b^{2}$, with $a$ and $|b|$ unique. If $b \equiv 0$ $(\bmod 4)($ so that $p \equiv 1(\bmod 8))$, Gauss [8: p. 89] (see also [4], [16]) has shown that

$$
2^{(p-1) / 4} \equiv \begin{cases}+1(\bmod p), & \text { if } b \equiv 0(\bmod 8) \\ -1(\bmod p), & \text { if } b \equiv 4(\bmod 8)\end{cases}
$$

If $b \equiv 2(\bmod 4)($ so that $p \equiv 5(\bmod 8))$, we can choose $b \equiv-2(\bmod 8)$, by changing the sign of $b$, if necessary, and Gauss [8: p. 89] (see also [4], [11: p. 66], [16]) has shown that

$$
2^{(p-1) / 4} \equiv-b / a(\bmod p)
$$

We note that $(-b / a)^{2} \equiv-1(\bmod \mathrm{p})$.

When $k=3$ and $p \equiv 1(\bmod 8)$, there are integers $a \equiv 1(\bmod 4)$ and $b \equiv 0(\bmod 4)$ such that $p=a^{2}+b^{2}$, with $a$ and $|b|$ unique. Now $\left\{2^{(p-1) / 8}\right\}^{4}=2^{(p-1) / 2} \equiv 1(\bmod p)$, as $p \equiv 1(\bmod 8)$, so $2^{(p-1) / 8}$ is a 4 th root of unity modulo $p$. If $b \equiv 0(\bmod 8)$, Reuschle [14] conjectured and Western [15] (see also [16]) proved that

$$
2^{(p-1) / 8} \equiv \begin{cases}(-1)^{(p-1) / 8}(\bmod p), & \text { if } b \equiv 0(\bmod 16), \\ (-1)^{(p+7) / 8}(\bmod p), & \text { if } b \equiv 8(\bmod 16) .\end{cases}
$$

If $b \equiv 4(\bmod 8)$, we can choose $b \equiv 4(-1)^{(p+7) / 8}(\bmod 16)$, by changing the sign of $b$, if necessary, and Lehmer [11: p. 70] has shown that

$$
2^{(p-1) / 8} \equiv-\frac{b}{a}(\bmod p) .
$$


It is the purpose of this paper to treat the cases $k=4$ and 5. For $k=4$ and $p \equiv 1(\bmod 16)$, there are integers $a \equiv 1(\bmod 4), b \equiv 0$ $(\bmod 4), c \equiv 1(\bmod 4), d \equiv 0(\bmod 2)$, such that $p=a^{2}+b^{2}=c^{2}$ $+2 d^{2}$, with $a,|b|, c,|d|$ unique. Now $\left\{2^{(p-1) / 16}\right\}^{8}=2^{(p-1) / 2} \equiv 1$ $(\bmod p)$, so $2^{(p-1) / 16}$ is an 8 th root of unity modulo $p$. Since

$$
\left\{\frac{-(a+b) d}{a c}\right\}^{2} \equiv-b / a(\bmod p)
$$

the 8 th roots of unity $(\bmod p)$ are given by $\{-(a+b) d / a c\}^{n}, n=$ $0,1, \ldots, 7$. Making use of a congruence due to Hasse [9: p. 232] (see also [5: Theorem 3], [17: p. 411]), we prove in $\$ 2$ the following extension of the criterion for 2 to be a 16th power $(\bmod p)$, which was conjectured by Cunningham [3: p. 88] and first proved by Aigner [1] (see also [16: p. 373]).

THEOREM 1. Let $p \equiv 1(\bmod 16)$ be a prime. Let $a \equiv 1(\bmod 4), b \equiv 0$ $(\bmod 4), c \equiv 1(\bmod 4), d \equiv 0(\bmod 2)$ be integers such that $p=a^{2}+b^{2}$ $=c^{2}+2 d^{2}$. It is well known that $b \equiv 0(\bmod 8) \Leftrightarrow d \equiv 0(\bmod 4)($ see for example [2: p. 68]). Then the values of $2^{(p-1) / 16}(\bmod p)$ are given in Table 1.

The case $b \equiv 0(\bmod 16)$ constitutes the criterion of CunninghamAigner.

For $k=5$ and $p \equiv 1(\bmod 32)$, there are integers $a \equiv 1(\bmod 4)$, $b \equiv 0(\bmod 4), c \equiv 1(\bmod 4), d \equiv 0(\bmod 2), x \equiv-1(\bmod 8), u \equiv v \equiv$ $w \equiv 0(\bmod 2)$, such that $p=a^{2}+b^{2}=c^{2}+2 d^{2}$ and

$$
\left\{\begin{array}{l}
p=x^{2}+2 u^{2}+2 v^{2}+2 w^{2} \\
2 x v=u^{2}-2 u w-w^{2}
\end{array}\right.
$$

with $a,|b|, c,|d|, x$ unique. If $(x, u, v, w)$ is a solution of (1.7), then all solutions are given by $\pm(x, u, v, w), \pm(x,-u, v,-w), \pm(x, w,-v,-u)$, $\pm(x,-w,-v, u)$ (see for example [12: p. 366]). Now $\left\{2^{(p-1) / 32}\right\}^{16}=$ $2^{(p-1) / 2} \equiv+1(\bmod p)$, so $2^{(p-1) / 32}$ is a 16 th root of unity modulo $p$.

Since

$$
\left\{\frac{(d x+c v)(a(u+w)-b(u-w))}{2 b d\left(u^{2}+w^{2}\right)}\right\}^{2} \equiv \frac{-(a+b) d}{a c} \quad(\bmod p)
$$

the 16 th roots of unity $(\bmod p)$ are given by

$$
\left\{\frac{(d x+c v)(a(u+w)-b(u-w))}{2 b d\left(u^{2}+w^{2}\right)}\right\}^{n}, \quad n=0,1, \ldots, 15 .
$$


Making use of another congruence due to Hasse [9: p. 233] (see also [7: eqn. (2)]), we prove in $\$ 3$ the following extension of the criterion for 2 to be a 32nd power $(\bmod p)$ due to Hasse [9: p. 232-238] and Evans [6: Theorem 7].

THEOREM 2. Let $p \equiv 1(\bmod 32)$ be a prime. Let $a \equiv 1(\bmod 4), b \equiv 0$ $(\bmod 4), c \equiv 1(\bmod 4), d \equiv 0(\bmod 2), x \equiv-1(\bmod 8), u \equiv v \equiv w \equiv 0$ $(\bmod 2)$, be integers such that $p=a^{2}+b^{2}=c^{2}+2 d^{2}$ and $p=x^{2}+$ $2 u^{2}+2 v^{2}+2 w^{2}, 2 x v=u^{2}-2 u w-w^{2}$. Then the values $2^{(p-1) / 32}$ $(\bmod p)$ are given in Table 2.

Justification of the choices in the left-hand column of Table 2 is made in the proof of Theorem 2, which appears in $\$ 3$. The cases $2^{(p-1) / 32} \equiv \pm 1$ $(\bmod p)$ constitute the criterion of Hasse-Evans.

2. Evaluation of $2^{(p-1) / 16}(\bmod p)$. Let $p$ be a prime satisfying

$$
p \equiv 1(\bmod 16) \text {. }
$$

Set

$$
p=8 f+1
$$

so that

$$
f \equiv 0(\bmod 2)
$$

Let

$$
\omega=\exp (2 \pi i / 8)=(1+i) / \sqrt{2} .
$$

We note that the ring of integers of $Q(\omega)=Q(i, \sqrt{2})$ is a unique factorization domain (see for example [13]). In this ring $p$ factors as a product of four primes. Denoting one of these by $\pi$, these four primes are $\pi_{j}=\sigma_{j}(\pi)$, $j=1,3,5,7$, where $\sigma_{j}$ denotes the automorphism which maps $\omega$ to $\omega^{j}$.

Let $g$ be a primitive root $(\bmod p)$. Then $g^{(p-1) / 2} \equiv-1(\bmod p)$, and so

$$
\left(g^{f}-\omega\right)\left(g^{f}-\omega^{3}\right)\left(g^{f}-\omega^{5}\right)\left(g^{f}-\omega^{7}\right) \equiv 0\left(\bmod \pi_{1} \pi_{3} \pi_{5} \pi_{7}\right) .
$$

Hence

$$
g^{f}-\omega^{j} \equiv 0\left(\bmod \pi_{1}\right),
$$

for some $j, j=1,3,5,7$, and by relabelling the $\pi$ 's we may assume without loss of generality that

$$
g^{f} \equiv \omega(\bmod \pi)
$$

Given $g, \pi$ (apart from units) is uniquely determined by (2.5). Next we define a character $\chi(\bmod p)$ (depending upon $g)$ of order 8 by setting

$$
\chi(g)=\omega .
$$


For $r, s=0,1,2, \ldots, 7$ the Jacobi $\operatorname{sum} J(r, s)$ is defined by

$$
J(r, s)=\sum_{n(\bmod p)} \chi^{r}(n) \chi^{s}(1-n) .
$$

It is known that (see for example [7: §1])

$$
J(2,2)=-a+b i,
$$

where

$$
p=a^{2}+b^{2}, \quad a \equiv 1(\bmod 4)
$$

and that

$$
J(1,3)=-c+d i \sqrt{2}
$$

where

$$
p=c^{2}+2 d^{2}, \quad c \equiv 1(\bmod 4) .
$$

It is easy to check that replacing the primitive root $g$ by the primitive root $g^{8 s+t}$, where $t=1,3,5,7$ and $(8 s+t, f)=1$, has the effect in (2.8) of replacing $b$ by $(-1 / t) b$ and in (2.10) of replacing $d$ by $(-2 / t) d$.

Our proof depends upon the following important congruence due to Hasse [9: p. 232]

$$
b \equiv 4 d+2 m(\bmod 32),
$$

where $m$ is the least positive integer such that

$$
g^{m} \equiv 2(\bmod p),
$$

and $b$ and $d$ are given by (2.8) and (2.10) respectively. From (2.12) and (2.13) we obtain

$$
2^{(p-1) / 16}=2^{f / 2} \equiv g^{m f / 2} \equiv g^{f(b / 4-d)}(\bmod p) .
$$

It follows from (2.5) and (2.6) that

$$
\chi(n) \equiv n^{f}(\bmod \pi),
$$

for any integer $n$ not divisible by $p$. Hence, for non-negative integers $r$ and $s$ satisfying $0 \leq r+s<8$, we have

$$
\begin{aligned}
J(r, s) & \equiv \sum_{n=0}^{p-1} n^{r f}(1-n)^{s f}(\bmod \pi) \\
& \equiv \sum_{n=0}^{p-1} n^{r f} \sum_{j=0}^{s f}\left(\begin{array}{c}
s f \\
j
\end{array}\right)(-1)^{j} n^{j}(\bmod \pi) \\
& \equiv \sum_{j=0}^{s f}\left(\begin{array}{c}
s f \\
j
\end{array}\right)(-1)^{j} \sum_{n=0}^{p-1} n^{r f+j}(\bmod \pi),
\end{aligned}
$$


that is

$$
J(r, s) \equiv 0(\bmod \pi)
$$

as

$$
\sum_{n=0}^{p-1} n^{k} \equiv 0(\bmod p), \text { for } k=0,1, \ldots, p-2 .
$$

Taking $(r, s)=(2,2)$ and $(1,3)$ in (2.16), we have, by (2.8) and (2.10),

$$
i \equiv a / b(\bmod \pi), i \sqrt{2} \equiv c / d(\bmod \pi)
$$

so that

$$
\sqrt{2} \equiv-a c / b d(\bmod \pi)
$$

Hence we have, appealing to (2.5), (2.18) and (2.19),

$$
g^{f} \equiv \omega=\frac{1+i}{\sqrt{2}} \equiv-\frac{(a+b) d}{a c}(\bmod \pi)
$$

and, since $g^{f}$ and $-(a+b) d / a c$ are integers $(\bmod p)$, we have

$$
g^{f} \equiv-\frac{(a+b) d}{a c}(\bmod p)
$$

Appealing to (2.14) we get

$$
2^{(p-1) / 16} \equiv\left\{\frac{-(a+b) d}{a c}\right\}^{(b / 4)-d}(\bmod p) .
$$

We consider three cases:

(i) $2^{(p-1) / 4} \equiv-1(\bmod p)$,

(ii) $2^{(p-1) / 4} \equiv+1,2^{(p-1) / 8} \equiv-1(\bmod p)$,

(iii) $2^{(p-1) / 8} \equiv+1(\bmod p)$.

Case (i). From $(1.2)$ we have $b \equiv 4(\bmod 8)$. Then, from $p=a^{2}+b^{2}$, we obtain $a \equiv 1(\bmod 8)$ and $p \equiv 2 a+15(\bmod 32)$. The cyclotomic number $(0,7)_{8}$ is given by (see for example [10: p. 116])

$$
64(0,7)_{8}=p-7+2 a+4 c,
$$

so $c \equiv 5(\bmod 8)$. Then, from $p=c^{2}+2 d^{2}$, we get $d \equiv 2(\bmod 4)$. Replacing $g$ by an appropriate primitive root

$$
g^{8 s+t}(t=1,3,5,7 ;(8 s+t, f)=1)
$$


we may take $b \equiv-4 \equiv 12(\bmod 16)$ and $d \equiv 2(\bmod 8)$. Then, from $(2.21)$, we obtain

$$
2^{(p-1) / 16} \equiv \begin{cases}-\frac{(a+b) d}{a c}(\bmod p), & \text { if } b \equiv 12(\bmod 32) \\ +\frac{(a+b) d}{a c}(\bmod p), & \text { if } b \equiv 28(\bmod 32)\end{cases}
$$

Case (ii). From (1.2) and (1.4) we have $b \equiv 8(\bmod 16)$. Then, from $p=a^{2}+b^{2}$, we obtain $a \equiv 1(\bmod 8)$ and $p \equiv 2 a-1(\bmod 32)$. The cyclotomic number $(1,2)_{8}$ is given by (see for example [10: p. 116])

$$
64(1,2)_{8}=p+1+2 a-4 c,
$$

so $c \equiv 1(\bmod 8)$. Then, from $p=c^{2}+2 d^{2}$, we get $d \equiv 0(\bmod 4)$. Replacing $g$ by an appropriate primitive root

$$
g^{8 s+t}(t=1,3 ;(8 s+t, f)=1)
$$

we may take $b \equiv 8(\bmod 32)$. Then as

$$
\left\{\frac{-(a+b) d}{a c}\right\}^{2} \equiv \frac{-b}{a}(\bmod p),
$$

we have from (2.21)

$$
2^{(p-1) / 16} \equiv \begin{cases}-b / a(\bmod p), & \text { if } d \equiv 0(\bmod 8) \\ +b / a(\bmod p), & \text { if } d \equiv 4(\bmod 8)\end{cases}
$$

Case (iii). From (1.4) we have $b \equiv 0(\bmod 16)$. Exactly as in Case (ii) we have $d \equiv 0(\bmod 4)$. Considering four cases according as $b \equiv$ $0,16(\bmod 32)$ and $d \equiv 0,4(\bmod 8)$ we obtain from $(2.21)$

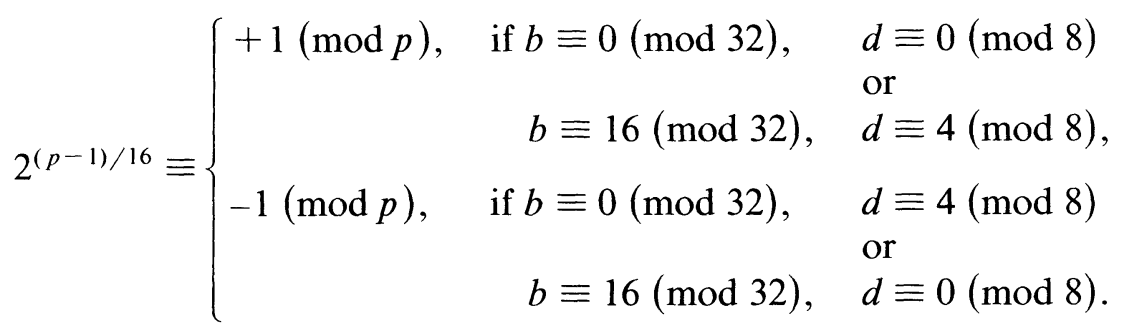

This completes the proof of Theorem 1 .

3. Evaluation of $2^{(p-1) / 32}(\bmod p)$. Let $p$ be a prime satisfying

$$
p \equiv 1(\bmod 32) \text {. }
$$


Set

$$
p=16 f+1
$$

so that

$$
f \equiv 0(\bmod 2)
$$

Let

$$
\theta=\exp (2 \pi i / 16)=\frac{1}{2}\{\sqrt{2+\sqrt{2}}+i \sqrt{2-\sqrt{2}}\} .
$$

Again, the ring of integers of $Q(\theta)$ is a unique factorization domain (see for example [13]). In this ring $p$ factors as a product of eight primes. Denoting one of these by $\pi$, these eight primes are given by $\pi_{i}=\sigma_{i}(\pi)$, $i=1,3,5,7,9,11,13,15$, where $\sigma_{i}$ denotes the automorphism which maps $\theta$ to $\theta^{i}$.

Let $g$ be a primitive $\operatorname{root}(\bmod p)$. Then

$$
\left(g^{f}-\theta\right)\left(g^{f}-\theta^{3}\right) \cdots\left(g^{f}-\theta^{15}\right) \equiv 0\left(\bmod \pi_{1} \pi_{3} \cdots \pi_{15}\right),
$$

and, as before, we can choose $\pi_{1}=\pi$ (unique apart from units) so that

$$
g^{f} \equiv \theta(\bmod \pi) \text {. }
$$

We define a character $\Psi(\bmod p)$ of order 16 by setting

$$
\Psi(g)=\theta,
$$

and for $r, s=0,1,2, \ldots, 15$ we define the Jacobi sum $J(r, s)$ by

$$
J(r, s)=\sum_{n(\bmod p)} \psi^{r}(n) \psi^{s}(1-n) .
$$

It is known that (see for example [7: §1])

$$
\begin{array}{ll}
J(4,4)=-a+b i, & \text { where } p=a^{2}+b^{2}, \quad a \equiv 1(\bmod 4), \\
J(2,6)=-c+d i \sqrt{2}, & \text { where } p=c^{2}+2 d^{2}, \quad c \equiv 1(\bmod 4),
\end{array}
$$

and

$$
\begin{aligned}
J(1,7) & =x+u i \sqrt{2-\sqrt{2}}+v \sqrt{2}+w i \sqrt{2+\sqrt{2}} \\
& =x+u\left(\theta+\theta^{7}\right)+v\left(\theta^{2}-\theta^{6}\right)+w\left(\theta^{3}+\theta^{5}\right),
\end{aligned}
$$

where (see for example [5; eqn. (8)])

$$
\left\{\begin{array}{l}
p=x^{2}+2 u^{2}+2 v^{2}+2 w^{2}, \quad x \equiv-1(\bmod 8), \\
2 x v=u^{2}-2 u w-w^{2}
\end{array}\right.
$$


It is easy to check that $u, v$ and $w$ are all even. Applying the mapping $\theta \rightarrow \theta^{3}$ to (3.10), we obtain

$$
\begin{aligned}
J(3,5) & =x-w i \sqrt{2-\sqrt{2}}-v \sqrt{2}+u i \sqrt{2+\sqrt{2}} \\
& =x-w\left(\theta+\theta^{7}\right)-v\left(\theta^{2}-\theta^{6}\right)+u\left(\theta^{3}+\theta^{5}\right) .
\end{aligned}
$$

Further, it is known (see [12: p. 366] and [6: eqn. (48)]) that $a, b, c, d, x, u$, $v, w$ are related by

$$
b d\left(x^{2}-2 v^{2}\right) \equiv a c\left(u^{2}+2 u w-w^{2}\right)(\bmod p) .
$$

The effect on (3.8), (3.9), (3.10) of replacing the primitive root $g$ by the primitive root $g^{16 s+t}$, where $t=1,3,5, \ldots, 15$ and $(16 s+t, f)=1$, is summarized below:

$$
\begin{array}{lllllllll}
g & a & b & c & d & x & u & v & w \\
g^{16 s+3} & a & -b & c & d & x & w & -v & -u \\
g^{16 s+5} & a & b & c & -d & x & w & -v & -u \\
g^{16 s+7} & a & -b & c & -d & x & u & v & w \\
g^{16 s+9} & a & b & c & d & x & -u & v & -w \\
g^{16 s+11} & a & -b & c & d & x & -w & -v & u \\
g^{16 s+13} & a & b & c & -d & x & -w & -v & u \\
g^{16 s+15} & a & -b & c & -d & x & -u & v & -w
\end{array}
$$

The following important congruence relating $b, d, u$ and $w$ has been proved by Hasse [9: p. 233]

$$
b+4 d-8(u+w) \equiv 2 m(\bmod 64),
$$

where $m$ satisfies (2.13). From (2.13) and (3.15), we obtain

$$
2^{(p-1) / 32}=2^{f / 2} \equiv g^{m f / 2} \equiv g^{f((b / 4)+d-2(u+w))}(\bmod p) .
$$

As in $\S 2$, if $r$ and $s$ are non-negative integers satisfying $0 \leq r+s<16$, we have

$$
J(r, s) \equiv 0(\bmod \pi) .
$$

Thus, in particular, taking $(r, s)=(4,4),(2,6),(1,7)$, and $(3,5)$, in $(3.17)$, we obtain

$$
\begin{gathered}
-a+b i \equiv 0(\bmod \pi), \\
-c+d i \sqrt{2} \equiv 0(\bmod \pi), \\
x+u i \sqrt{2-\sqrt{2}}+v \sqrt{2}+w i \sqrt{2+\sqrt{2}} \equiv 0(\bmod \pi), \\
x-w i \sqrt{2-\sqrt{2}}-v \sqrt{2}+u i \sqrt{2+\sqrt{2}} \equiv 0(\bmod \pi) .
\end{gathered}
$$


From (3.18) and (3.19) we get

$$
\begin{gathered}
i \equiv a / b \quad(\bmod \pi), \quad i \sqrt{2} \equiv c / d \quad(\bmod \pi), \\
\sqrt{2} \equiv-\frac{a c}{b d}(\bmod \pi) .
\end{gathered}
$$

Solving (3.20) and (3.21) simultaneously for $\sqrt{2+\sqrt{2}}$ and $\sqrt{2-\sqrt{2}}$ $(\bmod \pi)$, and making use of (3.22), we obtain

$$
\sqrt{2 \pm \sqrt{2}} \equiv \frac{x(u \pm w) a d \mp v(u \mp w) b c}{b d\left(u^{2}+w^{2}\right)}(\bmod \pi)
$$

Then, from (3.4), (3.5), (3.22) and (3.23), we have

$$
g^{f} \equiv \theta \equiv \frac{(d x+c v)(a(u+w)-b(u-w))}{2 b d\left(u^{2}+w^{2}\right)}(\bmod \pi) .
$$

Since both sides of (3.24) are integers $(\bmod p)$, we deduce that

$$
g^{f} \equiv \frac{(d x+c v)(a(u+w)-b(u-w))}{2 b d\left(u^{2}+w^{2}\right)}(\bmod p) .
$$

Appealing to (3.16) we get

$$
2^{(p-1) / 32} \equiv\left\{\frac{(d x+c v)(a(u+w)-b(u-w))}{2 b d\left(u^{2}+w^{2}\right)}\right\}^{(b / 4)+d-2(u+w)}
$$

$(\bmod p)$

We consider four cases:

(i) $2^{(p-1) / 4} \equiv-1(\bmod p)$,

(ii) $2^{(p-1) / 4} \equiv+1,2^{(p-1) / 8} \equiv-1(\bmod p)$,

(iii) $2^{(p-1) / 8} \equiv+1,2^{(p-1) / 16} \equiv-1(\bmod p)$,

(iv) $2^{(p-1) / 16} \equiv+1(\bmod p)$.

Case (i). From Case (i) of $\$ 2$ we have $b \equiv 4(\bmod 8)$ and $d \equiv 2$ $(\bmod 4)$. Next, from $(2.12)$ and (3.15), we obtain

$$
u+w \equiv d \equiv 2(\bmod 4) \text {, }
$$

so that

$$
(u, w) \equiv(0,2) \quad \text { or } \quad(2,0)(\bmod 4) .
$$

Replacing $g$ by an appropriate primitive root $g^{16 s+t}$ (where $t=1,3,5, \ldots$, 15 and $(16 s+t, f)=1)$, we can suppose that

(3.27) $b \equiv-4(\bmod 16), \quad u \equiv 0(\bmod 4), \quad w \equiv 2(\bmod 8)$. 
Exactly one 5-tuple $(b, d, u, v, w)$ satisfies (3.13) and (3.27). Then, from $2 x v=u^{2}-2 u w-w^{2}$, we obtain (recalling $\left.x \equiv-1(\bmod 8)\right)$

$$
v \equiv 2 \quad(\bmod 8)
$$

From the work of Evans and Hill [7: Table 2a], we have

$$
256\left\{(2,4)_{16}-(4,10)_{16}\right\}=32(v-d),
$$

so that, by (3.28),

$$
d \equiv v \equiv 2 \quad(\bmod 8) .
$$

The choice (3.27) makes the exponent $(b / 4)+d-2(u+w)$ in (3.26) congruent to $1(\bmod 4)$. We now consider cases according as $b \equiv 12,28$, 44, $60(\bmod 64) ; d \equiv 2,10(\bmod 16) ; u \equiv 0,4(\bmod 8)$. For example, if $b \equiv 12(\bmod 64), d \equiv 2(\bmod 16), u \equiv 0(\bmod 8)$, then $(b / 4)+d-$ $2(u+w) \equiv 1(\bmod 16)$, so that $(3.26)$ gives

(3.31) $2^{(p-1) / 32} \equiv \frac{(d x+c v)(a(u+w)-b(u-w))}{2 b d\left(u^{2}+w^{2}\right)}(\bmod p)$,

in this case. The other cases can be treated similarly, see Table 2 (VII).

Case (ii). From Case (ii) of $\S 2$, we have $b \equiv 8(\bmod 16)$ and $d \equiv 0$ (mod 4). Appealing to the work of Evans [5: Theorem 4 and its proof], we have

$$
\begin{array}{r}
u \equiv 2(\bmod 4), \quad v \equiv 4(\bmod 8), \quad w \equiv 2(\bmod 4) \\
\text { if } d \equiv 0(\bmod 8),
\end{array}
$$

and

$$
\begin{aligned}
& u \equiv 0(\bmod 4), \quad v \equiv 0(\bmod 8), \quad w \equiv 0(\bmod 4) \\
& \text { if } d \equiv 4(\bmod 8) .
\end{aligned}
$$

If $d \equiv 0(\bmod 8)$, replacing $g$ by $g^{16 s+t}$ (where $t=1,7,9,15$ and $(16 s+t, f)=1)$, as necessary, we can suppose that

$$
b \equiv 8(\bmod 32), \quad w \equiv 2(\bmod 8) .
$$

There are exactly two 5-tuples ( $b, d, u, v, w)$, which satisfy (3.13) and (3.34). These are

$$
(b, d, u, v, w) \text { and }(b,-d,-w,-v, u), \quad \text { if } u \equiv 2(\bmod 8),
$$
and

$$
(b, d, u, v, w) \text { and }(b,-d, w,-v,-u), \quad \text { if } u \equiv 6(\bmod 8) .
$$


We note that the 16th root of unity modulo $p$,

$$
\left\{\frac{(d x+c v)(a(u+w)-b(u-w))}{2 b d\left(u^{2}+w^{2}\right)}\right\}^{b / 4+d-2(u+w)},
$$

is independent of which 5-tuple is used, since

$$
\begin{gathered}
\left\{\frac{((-d) x+c(-v))(a(\mp w \pm u)-b(\mp w \mp u))}{2 b(-d)\left((\mp w)^{2}+( \pm u)^{2}\right)}\right\}^{(b / 4)-d-2(\mp w \pm u)} \\
=\left\{\frac{(d x+c v)(a(u+w)-b(u-w))}{2 b d\left(u^{2}+w^{2}\right)}\right\}^{A}
\end{gathered}
$$

where

$$
A= \begin{cases}13\left(\frac{b}{4}-d-2 u+2 w\right), & \text { if } u \equiv 2(\bmod 8) \\ 5\left(\frac{b}{4}-d+2 u-2 w\right), & \text { if } u \equiv 6(\bmod 8)\end{cases}
$$

moreover,

$$
\begin{array}{r}
13\left(\frac{b}{4}-d-2 u+2 w\right)-\left(\frac{b}{4}+d-2 u-2 w\right) \\
=3 b-14 d-24 u+28 w \equiv 0 \quad(\bmod 16) \\
5\left(\frac{b}{4}-d+2 u-2 w\right)-\left(\frac{b}{4}+d-2 u-2 w\right) \\
=b-6 d+12 u-8 w \equiv 0 \quad(\bmod 16)
\end{array}
$$

so that

$$
A \equiv \frac{b}{4}+d-2(u+w)(\bmod 16)
$$

The choice (3.34) makes the exponent $(b / 4)+d-2(u+w)$ in (3.26) congruent to $2(\bmod 8)$. We now consider cases according as $b \equiv$ $8,40(\bmod 64) ; d \equiv 0,8(\bmod 16) ; u \equiv 2,6(\bmod 8)$. For example if $b \equiv$ $8(\bmod 64), d \equiv 0(\bmod 16), u \equiv 6(\bmod 8)$, then $(b / 4)+d-$ $2(u+w) \equiv 2(\bmod 16)$, so (3.26) gives

$$
\begin{aligned}
2^{(p-1) / 32} & \equiv\left\{\frac{(d x+c v)(a(u+w)-b(u-w))}{2 b d\left(u^{2}+w^{2}\right)}\right\}^{2}(\bmod p) \\
& \equiv \frac{-(a+b) d}{a c}(\bmod p)
\end{aligned}
$$


see Table 2(VI). We remark that in applying Theorem 2 in this case, $d$ must be chosen to satisfy the congruence (3.13). We can do this as $x^{2}-2 v^{2} \neq 0(\bmod p)$, since

$$
-p=-x^{2}-2 u^{2}-2 v^{2}-2 w^{2}<x^{2}-2 v^{2} \leq x^{2}<p .
$$

If $d \equiv 4(\bmod 8)$, replacing $g$ by $g^{16 s+t}$ (where $t=1,3,5$ or 7 and $(16 s+t, f)=1)$, as necessary, we can suppose that

$$
b \equiv-8 \equiv 24(\bmod 32), \quad d \equiv 4(\bmod 16) \text {. }
$$

There are precisely two 5 -tuples $(b, d, u, v, w)$, which satisfy (3.13) and (3.36). These are

$$
(b, d, u, v, w) \text { and }(b, d,-u, v,-w) .
$$

We note that the 16 th root of unity modulo $p$,

$$
\left\{\frac{(d x+c v)(a(u+w)-b(u-w))}{2 b d\left(u^{2}+w^{2}\right)}\right\}^{(b / 4)+d-2(u+w)}
$$

is independent of which 5-tuple is chosen, since

$$
\begin{gathered}
\left\{\frac{(d x+c v)(a(-u-w)-b(-u+w))}{2 b d\left((-u)^{2}+(-w)^{2}\right)}\right\}^{(b / 4)+d-2(-u-w)} \\
=\left\{\frac{(d x+c v)(a(u+w)-b(u-w))}{2 b d\left(u^{2}+w^{2}\right)}\right\}^{B},
\end{gathered}
$$

where

$$
B=9\left(\frac{b}{4}+d+2 u+2 w\right) \equiv \frac{b}{4}+d-2(u+w)(\bmod 16) .
$$

The choice (3.36) makes the component $(b / 4)+d-2(u+w)$ in (3.26) congruent to $2(\bmod 8)$. We now consider cases according as $b \equiv 24,56$ $(\bmod 64) ; u+w \equiv 0,4(\bmod 8)$. For example, if $b \equiv 56(\bmod 64)$, $u+w \equiv 4(\bmod 8)$, then $(b / 4)+d-2(u+w) \equiv 10(\bmod 16)$, so $(3.26)$ gives

$$
\begin{aligned}
2^{(p-1) / 32} & \equiv\left\{\frac{(d x+c v)(a(u+w)-b(u-w))}{2 b d\left(u^{2}+w^{2}\right)}\right\}^{10}(\bmod p) \\
& \equiv\left\{\frac{-(a+b) d}{a c}\right\}^{5}(\bmod p) \\
& \equiv \frac{+(a+b) d}{a c}(\bmod p)
\end{aligned}
$$


see Table 2(V). However, when applying Theorem 2 in this case, it is not necessary to use the congruence $b d\left(x^{2}-2 v^{2}\right) \equiv a c\left(u^{2}+2 u w-w^{2}\right)$ $(\bmod p)$ to distinguish the solutions $(x, \pm u, v, \pm w)$ from the solutions $(x, \pm w,-v, \mp u)$. since $\pm w \mp u \equiv \pm(u+w)(\bmod 8)$, as $u \equiv w \equiv 0$ $(\bmod 4)$.

Case (iii) From Case (iii) of $\$ 2$ we have

$$
b \equiv 0(\bmod 32), \quad d \equiv 4(\bmod 8),
$$

or

$$
b \equiv 16(\bmod 32), \quad d \equiv 0(\bmod 8) .
$$

If $b \equiv 0(\bmod 32), d \equiv 4(\bmod 8)$, from the work of Evans [5: Theorem 4 and its proof], we have

$$
u \equiv 2(\bmod 4), \quad v \equiv 4(\bmod 8), w \equiv 2(\bmod 4) .
$$

Replacing $g$ by $g^{16 s+t}$, where $t=1,7,9$ or 15 and $(16 s+t, f)=1$, as necessary, we can suppose that

$$
d \equiv 4(\bmod 16), w \equiv 2(\bmod 8) .
$$

There are exactly two 5-tuples $(b, d, u, v, w)$ which satisfy (3.13) and (3.41). These are

$$
(b, d, u, v, w) \text { and }(-b, d,-w,-v, u), \quad \text { if } u \equiv 2(\bmod 8),
$$

and

$$
(b, d, u, v, w) \text { and }(-b, d, w,-v,-u), \quad \text { if } u \equiv 6(\bmod 8)
$$

We note that the 16 th root of unity modulo $p$,

$$
\left\{\frac{(d x+c v)(a(u+w)-b(u-w))}{2 b d\left(u^{2}+w^{2}\right)}\right\}^{(b / 4)+d-2(u+w)}
$$

is independent of which 5-tuple is used, since

$$
\begin{gathered}
\left\{\frac{(d x+c(-v))(a(\mp w \pm u)+b(\mp w \mp u))}{\left.2(-b) d\left((\mp w)^{2}+(\mp u)^{2}\right)\right)}\right\}^{(-b / 4)+d-2(\mp w \pm u)} \\
=\left\{\frac{(d x+c v)(a(u+w)-b(u-w))}{2 b d\left(u^{2}+w^{2}\right)}\right\}^{C}
\end{gathered}
$$


where

$$
C= \begin{cases}11\left(-\frac{b}{4}+d-2 u+2 w\right), & \text { if } u \equiv 2(\bmod 8), \\ 3\left(-\frac{b}{4}+d+2 u-2 w\right), & \text { if } u \equiv 6(\bmod 8),\end{cases}
$$

and it is easily checked that

$$
C \equiv \frac{b}{4}+d-2(u+w) \quad(\bmod 16) .
$$

Clearly, from (3.38) and (3.40), we have $(b / 4)+d-2(u+w) \equiv 4$ $(\bmod 8)$, and we determine $(b / 4)+d-2(u+w)(\bmod 16)$ by considering the cases $b \equiv 0,32(\bmod 64)$ and $u \equiv 2,6(\bmod 8)$. For example, if $b \equiv 0(\bmod 64)$ and $u \equiv 6(\bmod 8)$, we have $(b / 4)+d-2(u+w) \equiv 4$ $(\bmod 16)$, so by $(3.26),(1.6)$ and (1.8),

$$
\begin{aligned}
2^{(p-1) / 32} & \equiv\left\{\frac{(d x+c v)(a(u+w)-b(u-w))}{2 b d\left(u^{2}+w^{2}\right)}\right\}^{4} \\
& \equiv-\frac{b}{a}(\bmod p)
\end{aligned}
$$

see Table 2 (III). In applying Theorem 2 in this case we must use the congruence $b d\left(x^{2}-2 v^{2}\right) \equiv a c\left(u^{2}+2 u w-w^{2}\right)(\bmod p)$ to distinguish the solutions $(x, \pm u, v, \pm w)$ from the solutions $(x, \mp w,-v, \pm u)$.

If $b \equiv 16(\bmod 32), d \equiv 0(\bmod 8)$, from the work of Evans [5: Theorem 4 and its proof], we have

$$
u \equiv 0(\bmod 4), \quad v \equiv 0(\bmod 8), \quad w \equiv 0(\bmod 4)
$$

Replacing $g$ by $g^{16 s+t}$, where $t=1$ or 7 and $(16 s+t, f)=1$, as necessary, we may suppose that

$$
b \equiv 16(\bmod 64) \text {. }
$$

There are exactly four 5-tuples $(b, d, u, v, w)$, which satisfy (3.13) and (3.44). These are

$$
(b, d, \pm u, v, \pm w), \quad(b,-d, \pm w,-v, \mp u) .
$$

We note as before that the 16 th root of unity modulo $p$,

$$
\left\{\frac{(d x+c v)(a(u+w)-b(u-w))}{2 b d\left(u^{2}+w^{2}\right)}\right\}^{(b / 4)+d-2(u+w)}
$$

is independent of which 5-tuple is used. 
Clearly, from (3.39) and (3.43), we have (b/4) $+d-2(u+w) \equiv 4$ $(\bmod 8)$, and we determine $(b / 4)+d-2(u+w)(\bmod 16)$ by considering the cases $d \equiv 0,8(\bmod 16)$ and $u+w \equiv 0,4(\bmod 8)$. For example, if $d \equiv 0(\bmod 16)$ and $u+w \equiv 4(\bmod 8)$, then $(b / 4)+d-2(u+w) \equiv 12$ $(\bmod 16)$, so by $(3.26),(1.6)$ and (1.8),

$$
\begin{aligned}
2^{(p-1) / 32} & \equiv\left\{\frac{(d x+c v)(a(u+w)-b(u-w))}{2 b d\left(u^{2}+w^{2}\right)}\right\}^{12} \\
& \equiv+\frac{b}{a}(\bmod p),
\end{aligned}
$$

see Table 2(IV). When applying Theorem 2 in this case, we can use any one of the four solutions $(x, \pm u, v, \pm w),(x, \pm w,-v, \mp u)$, as $\pm w \mp u \equiv \pm(u+w)(\bmod 8)$.

Case (iv). As $2^{(p-1) / 16} \equiv 1(\bmod p)$, from Table 1 , we have

$$
b \equiv 0(\bmod 32), \quad d \equiv 0(\bmod 8),
$$

or

$$
b \equiv 16(\bmod 32), \quad d \equiv 4(\bmod 8) .
$$

If $b \equiv 0(\bmod 32), d \equiv 0(\bmod 8)$, appealing to the work of Evans [5: Theorem 4 and its proof], we have

$$
u \equiv 0(\bmod 4), \quad v \equiv 0(\bmod 8), \quad w \equiv 0(\bmod 4) .
$$

There are exactly eight 5-tuples which satisfy (3.13) and (3.48), namely,

$$
\begin{gathered}
(b, d, \pm u, v, \pm w), \quad(b,-d, \pm w,-v, \mp u), \\
(-b, d, \pm w,-v, \mp u), \quad(-b,-d, \pm u, v, \pm w) .
\end{gathered}
$$

It is straightforward to check that

$$
\left\{\frac{(d x+c v)(a(u+w)-b(u-w))}{2 b d\left(u^{2}+w^{2}\right)}\right\}^{(b / 4)+d-2(u+w)}
$$

is the same for all of these. The exponent $(b / 4)+d-2(u+w)$ is congruent to $0(\bmod 8)$. It is easily determined modulo 16 by considering the cases $b \equiv 0,32(\bmod 64), d \equiv 0,8(\bmod 16)$, and $u+w \equiv 0,4$ $(\bmod 8)$. For example, if $b \equiv 0(\bmod 64), d \equiv 0(\bmod 16), u+w \equiv 4$ $(\bmod 8)$, we have $b / 4+d-2(u+w) \equiv 8(\bmod 16)$ so that, by $(1.6)$, (1.8) and (3.26),

$$
2^{(p-1) / 32} \equiv\left\{\frac{(d x+c v)(a(u+w)-b(u-w))}{2 b d\left(u^{2}+w^{2}\right)}\right\}^{8} \equiv-1(\bmod p)
$$


see Table 2 (I). As noted by Evans [6: Comments following Theorem 7], it is unnecessary to use the congruence $b d\left(x^{2}-2 v^{2}\right) \equiv a c\left(u^{2}+2 u w-w^{2}\right)$ $(\bmod p)$ when applying Theorem 2 in this case.

Finally if $b \equiv 16(\bmod 32), d \equiv 4(\bmod 8)$, appealing to the work of Evans [5: Theorem 4 and its proof], we have

$$
u \equiv 2(\bmod 4), \quad v \equiv 4(\bmod 8), \quad w \equiv 2(\bmod 4) \text {. }
$$

Replacing $g$ by $g^{16 s+t}$, where $t=1,3,5$ or 7 and $(16 s+t, f)=1$, as appropriate, we can choose

$$
b \equiv 16(\bmod 64), \quad d \equiv 4(\bmod 16) .
$$

There are two 5-tuples ( $b, d, u, v, w)$ satisfying (3.13) and (3.49), namely,

$$
(b, d, \pm u, v, \pm w)
$$

and again it is easy to check that

$$
\begin{gathered}
\left\{\frac{(d x+c v)(a(u+w)-b(u-w))}{2 b d\left(u^{2}+w^{2}\right)}\right\}^{(b / 4)+d-2(u+w)} \\
=\left\{\frac{(d x+c v)(a(-u-w)-b(-u+w))}{2 b d\left((-u)^{2}+(-w)^{2}\right)}\right\}^{(b / 4)+d-2(-u-w)} .
\end{gathered}
$$

Now

$$
\frac{b}{4}+d-2(u+w) \equiv 8-2(u+w)(\bmod 16)
$$

so, by (3.26), we have

$$
2^{(p-1) / 32} \equiv \begin{cases}+1, & \text { if } u+w \equiv 4(\bmod 8) \\ -1, & \text { if } u+w \equiv 0(\bmod 8)\end{cases}
$$

see Table 2 (II). In applying Theorem 2 in this case, as noted by Evans [6: Comments following Theorem 7], it is necessary to use the congruence $b d\left(x^{2}-2 v^{2}\right) \equiv a c\left(u^{2}+2 u w-w^{2}\right)(\bmod p)$. This completes the proof of Theorem 2 .

4. Numerical examples. (a) $p=2113$ (see Table 2 (I)). We have

$$
\begin{gathered}
(a, b)=(33, \pm 32) ; \quad a \equiv 1(\bmod 4) ; \\
(c, d)=(-31, \pm 24) ; \quad c \equiv 1(\bmod 4) ; \\
(x, u, v, w)=(-17, \pm 28,-8, \pm 8) \text { or }(-17, \pm 8,+8, \mp 28) ; \\
x \equiv-1 \quad(\bmod 8) .
\end{gathered}
$$


For each choice we have

$$
b \equiv 32(\bmod 64), \quad d \equiv 8(\bmod 16), \quad u+w \equiv 4(\bmod 8),
$$

so by Theorem 2(I), we have

$$
2^{(p-1) / 32}=2^{66} \equiv-1 \quad(\bmod 2113) .
$$

(b) $p=257$ (see Table 2 (II)). We have

$$
\begin{gathered}
(a, b)=(1,16) ; \quad a \equiv 1(\bmod 4), \quad b \equiv 16(\bmod 64) ; \\
(c, d)=(-15,4) ; \quad c \equiv 1(\bmod 4), \quad d \equiv 4(\bmod 16) ; \\
(x, u, v, w)=(-9, \pm 6,-4, \mp 6) \text { or } \quad(-9, \pm 6,+4, \pm 6) ; \\
x \equiv-1(\bmod 8) .
\end{gathered}
$$

The congruence $b d\left(x^{2}-2 v^{2}\right) \equiv a c\left(u^{2}+2 u w-w^{2}\right)(\bmod p)$ is satisfied by $(x, u, v, w)=(-9, \pm 6,-4, \mp 6)$. As $u+w \equiv 0(\bmod 8)$, by Theorem 2(II), we have

$$
2^{(p-1) / 32}=2^{8} \equiv-1(\bmod 257) .
$$

(c) $p=1249$ (see Table 2(III)). We have

$$
\begin{gathered}
(a, b)=(-15,32) \text { or }(-15,-32) ; \\
a \equiv 1(\bmod 4), \quad b \equiv 0(\bmod 32) ; \\
(c, d)=(-31,-12) ; \quad c \equiv 1(\bmod 4), \quad d \equiv 4(\bmod 16) ; \\
(x, u, v, w)=(7,10,4,-22) \quad \text { or }(7,22,-4,10) ; \\
x \equiv-1(\bmod 8), \quad w \equiv 2(\bmod 8) .
\end{gathered}
$$

The congruence $b d\left(x^{2}-2 v^{2}\right) \equiv a c\left(u^{2}+2 u w-w^{2}\right)(\bmod p)$ is satisfied by $(a, b)=(-15,32)$ and $(x, u, v, w)=(7,22,-4,10)$ or by $(a, b)=(-15,-32)$ and $(x, u, v, w)=(7,10,4,-22)$. Hence, by Theorem 2 , taking $b=32, u=22 \equiv 6(\bmod 8)$, we have

$$
2^{(p-1) / 32}=2^{39} \equiv+b / a \equiv 32 /-15 \equiv 664(\bmod 1249) ;
$$

taking $b=-32, u=10 \equiv 2(\bmod 8)$, we have

$$
2^{(p-1) / 32}=2^{39} \equiv-b / a \equiv 32 /-15 \equiv 664(\bmod 1249) .
$$

(d) $p=1217$ (see Table 2 (IV)). We have

$$
\begin{gathered}
(a, b)=(-31,16) ; \quad a \equiv 1(\bmod 4), \quad b \equiv 16(\bmod 64) ; \\
(c, d)=(33,+8) \text { or }(33,-8) ; \quad c \equiv 1(\bmod 4) ; \\
(x, u, v, w)=(-17, \pm 12,-8, \mp 16), \quad(-17, \pm 16,+8, \pm 12) \\
x \equiv-1(\bmod 8) .
\end{gathered}
$$


As $d \equiv 8(\bmod 16)$ and $u+w \equiv 4(\bmod 8)$ (for each possibility), we have, by Theorem 2 ,

$$
2^{(p-1) / 32}=2^{38} \equiv-b / a \equiv 16 / 31 \equiv 1139(\bmod 1217) .
$$

(e) $p=577$ (see Table $2(\mathrm{~V}))$. We have

$$
\begin{gathered}
(a, b)=(1,24) ; \quad a \equiv 1(\bmod 4), \quad b \equiv 24(\bmod 32) ; \\
(c, d)=(17,-12) ; \quad c \equiv 1(\bmod 4), \quad d \equiv 4(\bmod 16) ; \\
(x, u, v, w)=(-1, \pm 4,-16, \mp 4) \text { or }(-1, \pm 4,+16, \pm 4) .
\end{gathered}
$$

As $b \equiv 24(\bmod 64), u+w \equiv 0(\bmod 8)$, by Theorem $2(\mathrm{~V})$, we have

$$
2^{(p-1) / 32}=2^{18} \equiv+\frac{(a+b) d}{a c} \equiv \frac{-300}{17} \equiv 186(\bmod 577) .
$$

(f) $p=353$ (see Table $2(\mathrm{VI})$ ). We have

$$
\begin{gathered}
(a, b)=(17,8) ; \quad a \equiv 1(\bmod 4), \quad b \equiv 8(\bmod 32) ; \\
(c, d)=(-15,8) \text { or }(-15,-8) ; \quad c \equiv 1(\bmod 4) ; \\
(x, u, v, w)=(7,-10,-4,-6) \text { or }(7,-6,4,10) ; \\
x \equiv-1(\bmod 8), \quad w \equiv 2(\bmod 8) .
\end{gathered}
$$

The congruence $b d\left(x^{2}-2 v^{2}\right) \equiv a c\left(u^{2}+2 u w-w^{2}\right)(\bmod p)$ is satisfied by $(c, d)=(-15,8)$ and $(x, u, v, w)=(7,-10,-4,-6)$, or by $(c, d)=$ $(-15,-8)$ and $(x, u, v, w)=(7,-6,4,10)$. Hence, by Theorem 2 , taking the first possibility, we have $b \equiv 8(\bmod 64), d \equiv 8(\bmod 16), u=-10 \equiv 6$ $(\bmod 8)$, so

$$
2^{(p-1) / 32}=2^{11} \equiv \frac{(a+b) d}{a c} \equiv \frac{40}{-51} \equiv 283(\bmod 353) .
$$

(g) $p=97$ (see Table 2 (VIII)). We have

$$
\begin{gathered}
(a, b)=(9,-4) ; \quad a \equiv 1(\bmod 4), \quad b \equiv 12(\bmod 16) ; \\
(c, d)=(5,-6) ; \quad c \equiv 1(\bmod 4), \quad d \equiv 2(\bmod 8) ; \\
(x, u, v, w)=(7,-4,2,2) ; \quad x \equiv-1(\bmod 8), \quad w \equiv 2(\bmod 8) .
\end{gathered}
$$

As $b \equiv 60(\bmod 64), d \equiv 10(\bmod 16), u \equiv 4(\bmod 8)$, by Theorem $2(\mathrm{VII})$, we have

$$
2^{(p-1) / 32}=2^{3} \equiv \frac{(-32)(-46)}{(48)(20)} \equiv \frac{23}{15} \equiv 8(\bmod 97) .
$$

5. Acknowledgement. We wish to thank Mr. Lee-Jeff Bell for doing some calculations for us in connection with the preparation of this paper. 


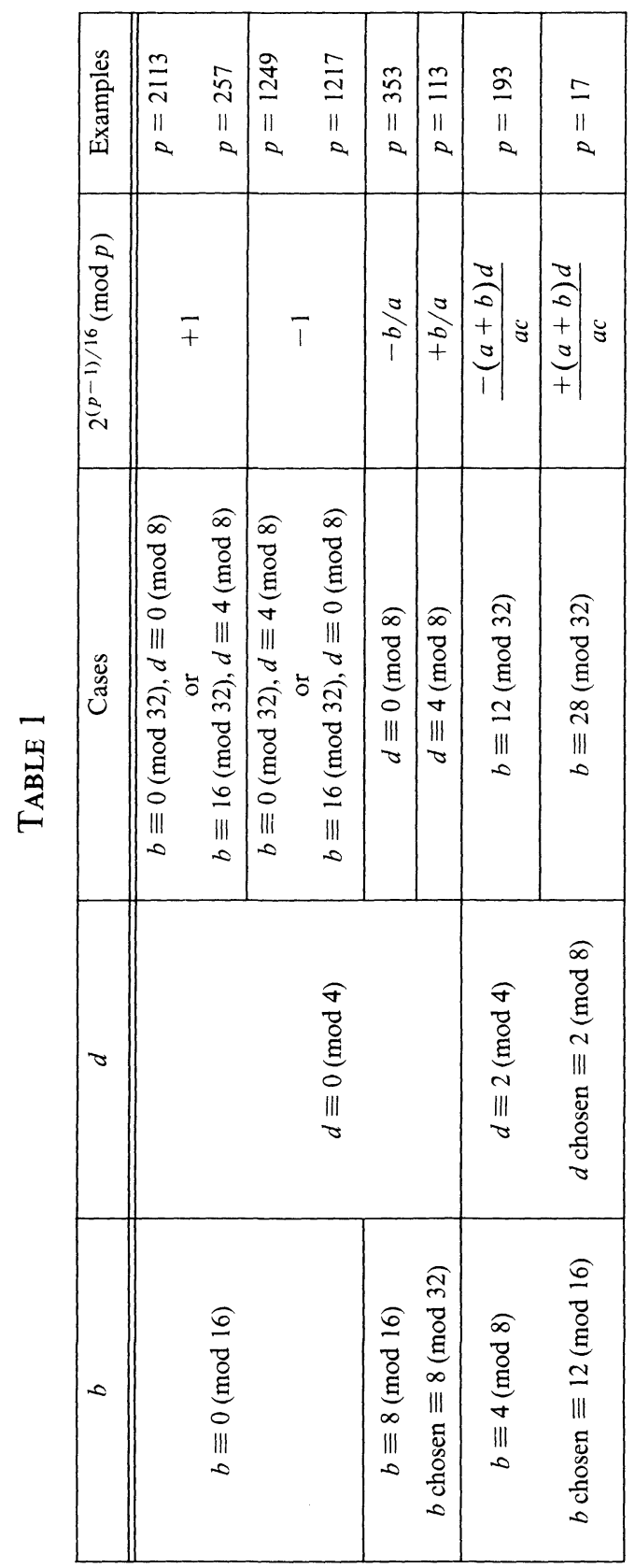




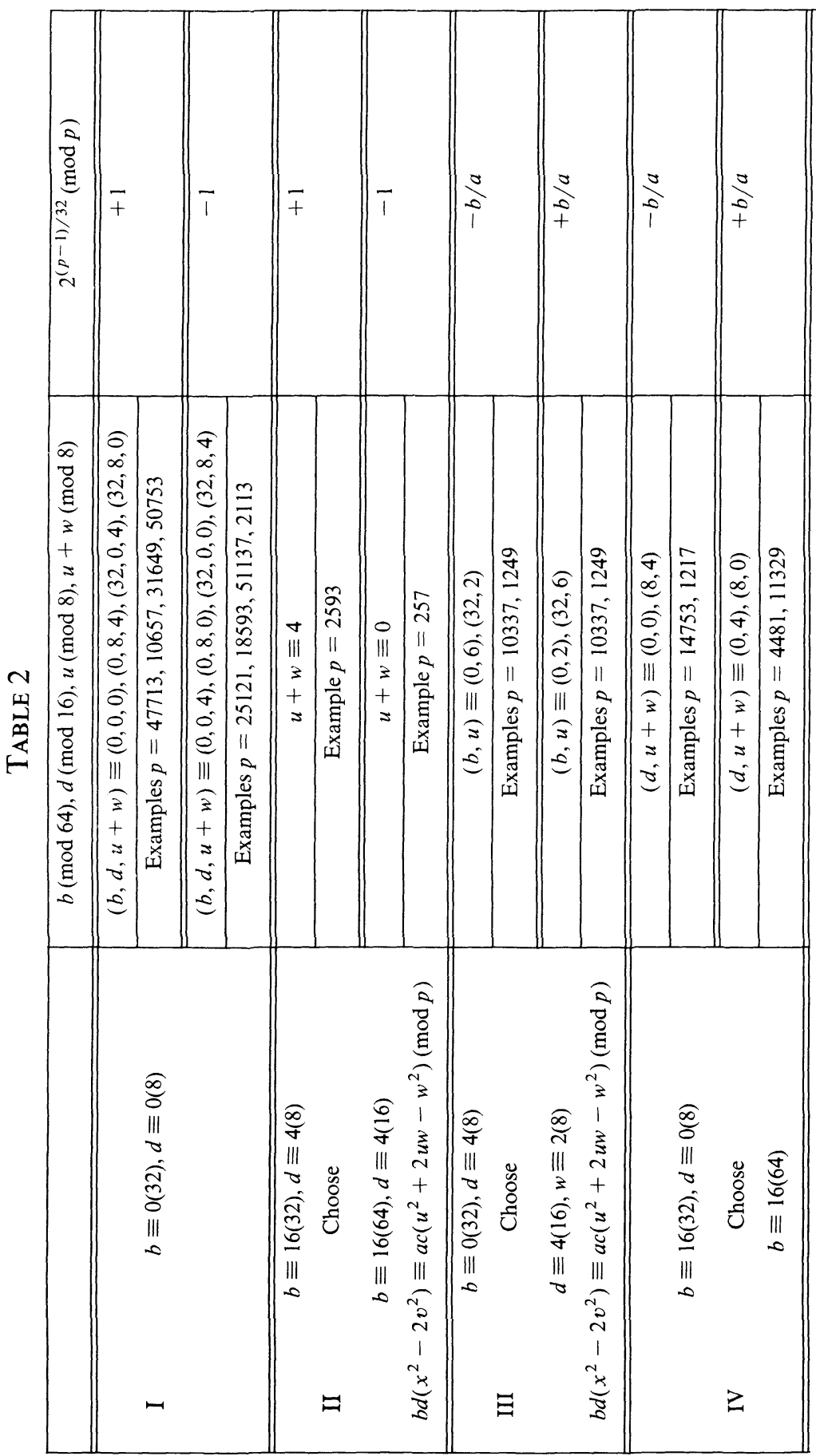




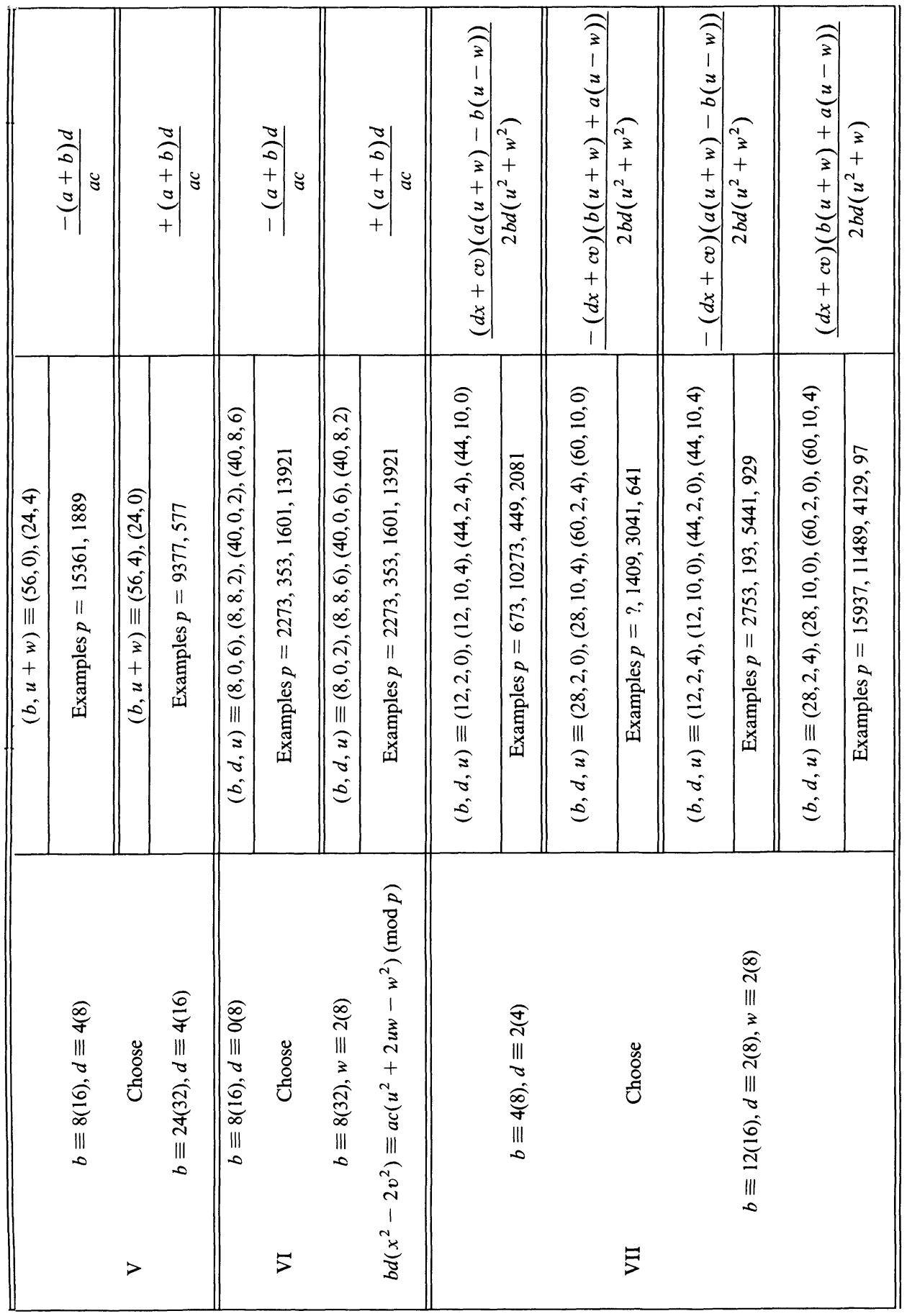




\section{REFERENCES}

1. A. Aigner, Kriterien zum 8. und 16. Potenzcharakter der Reste 2 und -2, Deutsche Math., 4 (1939), 44-52.

2. P. Barrucand and H. Cohn, Note on primes of type $x^{2}+32 y^{2}$, class number, and residuacity, J. Reine Angew. Math., 238 (1969), 67-70.

3. A. Cunningham, On 2 as a 16-ic residue, Proc. London Math. Soc., (1) 27 (1895/6), 85-122.

4. G. L. Dirichlet, Ueber den biquadratischen Charakter der Zahl "Zwei", J. Reine Angew. Math., 57 (1860), 187-188.

5. R. J. Evans, Resolution of sign ambiguities in Jacobi and Jacobsthal sums, Pacific J. Math., 81 (1979), 71-80.

6. The $2^{r}$ th power character of 2, J. Reine Angew. Math., 315 (1980), 174-189.

7. R. J. Evans and J. R. Hill, The cyclotomic numbers of order sixteen, Math. Comp., 33 (1979), 827-835.

8. C. F. Gauss, Theoria residuorum biquadraticorum. I, Commentationes soc. reg. sc. Gotting. recentiores., 6 (1828), 27- . II. 7 (1832), 89- , (See Untersuchungen über Höhere Arithmetik, Chelsea Publ. Co. (N. Y.) (1965), 511-586.)

9. H. Hasse, Der $2^{n}$ te Potenzcharakter von 2 im Körper der $2^{n}$ ten Einheitswurzeln, Rend. Circ. Mat. Palermo, Serie II, 7 (1958), 185-244.

10. Emma Lehmer, On the number of solutions of $u^{k}+D \equiv w^{2}(\bmod p)$, Pacific J. Math., 5 (1955), 103-118.

11. On Euler's criterion, J. Austral. Math. Soc., 1 (1959), 64-70.

12. P. A. Leonard and K. S. Williams, A rational sixteenth power reciprocity law, Acta Arith., 33 (1977), 365-377.

13. J. M. Masley and H. L. Montgomery, Cyclotomic fields with unique factorization, J. Reine Angew. Math., 286/287 (1976), 248-256.

14. C. G. Reuschle, Mathematische Abhandlung, enthaltend neue Zahlentheoretische Tabellen, Programm zum Schlusse des Schuljahrs 1855-56 am Königlichen Gymnasium zu Stuttgart (1856), $61 \mathrm{pp}$.

15. A. E. Western, Some criteria for the residues of eighth and other powers, Proc. London Math., (2), 9 (1911), 244-272.

16. A. L. Whiteman, The sixteenth power residue character of 2, Canad. J. Math., 6 (1954), 364-373.

17. The cyclotomic numbers of order sixteen, Trans. Amer. Math. Soc., 86 (1957), 401-413.

Received November 19, 1979 and in revised form August 18, 1981. Research supported by grant no. A-7233 of the Natural Sciences and Engineering Research Council Canada.

University of South Carolina

COLUMBIA, SC U.S.A 29208

AND

CARLETON UNIVERSiTY

OtTawa, Ontario, Canada K1S 5B6 


\section{PACIFIC JOURNAL OF MATHEMATICS \\ EDITORS}

DONALD BABBITT (Managing Editor)

University of California

Los Angeles, CA 90024

\section{Hugo Rossi}

University of Utah

Salt Lake City, UT 84112

C. C. Moore and Arthur Ogus

University of California

Berkeley, CA 94720
J. DugundiI

Department of Mathematics

University of Southern California

Los Angeles, CA 90089-1113

R. FINN and H. SAMELSON

Stanford University

Stanford, CA 94305

ASSOCIATE EDITORS
R. ARens
E. F. BECKENBACH
B. H. NeumanN
F. WOLF
K. YoshidA (1906-1982)

\section{SUPPORTING INSTITUTIONS}

UNIVERSITY OF ARIZONA

UNIVERSITY OF BRITISH COLUMBIA

CALIFORNIA INSTITUTE OF TECHNOLOGY

UNIVERSITY OF CALIFORNIA

MONTANA STATE UNIVERSITY

UNIVERSITY OF NEVADA, RENO

NEW MEXICO STATE UNIVERSITY

OREGON STATE UNIVERSITY
UNIVERSITY OF OREGON

UNIVERSITY OF SOUTHERN CALIFORNIA

STANFORD UNIVERSITY

UNIVERSITY OF HAWAII

UNIVERSITY OF TOKYO

UNIVERSITY OF UTAH

WASHINGTON STATE UNIVERSITY

UNIVERSITY OF WASHINGTON 


\section{Pacific Journal of Mathematics}

Vol. 104, No. 1

May, 1983

Nestor Edgardo Aguilera and Eleonor Ofelia Harboure de Aguilera, On

the search for weighted norm inequalities for the Fourier transform $\ldots \ldots .1$

Jin Akiyama, Frank Harary and Phillip Arthur Ostrand, A graph and its complement with specified properties. VI. Chromatic and achromatic numbers ......................................... 15

Bing Ren Li, The perturbation theory for linear operators of discrete type . . . 29

Peter Botta, Stephen J. Pierce and William E. Watkins, Linear

transformations that preserve the nilpotent matrices .............. 39

Frederick Ronald Cohen, Ralph Cohen, Nicholas J. Kuhn and Joseph

Alvin Neisendorfer, Bundles over configuration spaces .......... 47

Luther Bush Fuller, Trees and proto-metrizable spaces . . . . . . . . . . 55

Giovanni P. Galdi and Salvatore Rionero, On the best conditions on the

gradient of pressure for uniqueness of viscous flows in the whole space . . 77

John R. Graef, Limit circle type results for sublinear equations $\ldots \ldots \ldots \ldots 85$

Andrzej Granas, Ronald Bernard Guenther and John Walter Lee,

Topological transversality. II. Applications to the Neumann problem for

$y^{\prime \prime}=f\left(t, y, y^{\prime}\right) \ldots \ldots \ldots \ldots \ldots \ldots \ldots \ldots \ldots \ldots \ldots \ldots \ldots \ldots \ldots . \ldots 5$

Richard Howard Hudson and Kenneth S. Williams, Extensions of

theorems of Cunningham-Aigner and Hasse-Evans . . . . . . . . . . . 111

John Francis Kurtzke, Jr., Centralizers of irregular elements in reductive algebraic groups

James F. Lawrence, Lopsided sets and orthant-intersection by convex

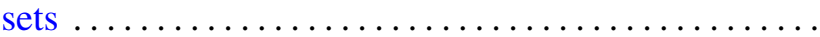

Åsvald Lima, G. H. Olsen and U. Uttersrud, Intersections of $M$-ideals and

$G$-spaces

Wallace Smith Martindale, III and C. Robert Miers, On the iterates of derivations of prime rings

Thomas H. Pate, Jr, A characterization of a Neuberger type iteration procedure that leads to solutions of classical boundary value problems

Carl L. Prather and Ken Shaw, Zeros of successive iterates of multiplier-sequence operators

Billy E. Rhoades, The fine spectra for weighted mean operators

Rudolf J. Taschner, A general version of van der Corput's difference theorem

Johannes A. Van Casteren, Operators similar to unitary or selfadjoint ones 